\title{
Mattress Overlapping Recognition Based on Markov Random Field
}

\author{
Fengyu $\mathrm{Hu}^{*}$ and Xianqiao Chen \\ No. 1178 Heping Avenue, Wuchang District, Hubei, Wuhan, China \\ ${ }^{*}$ Corresponding author
}

\begin{abstract}
Mattress overlapping recognition is of great significance in tracing and detecting the quality, and provides reference data of mattress laying. The recognition process includes the generation and image correction of the color sinking sonar image, the image segmentation and the recognition of mattress overlapping; In which the key issue is the segmentation of sonar image segmentation. There are a lot of underwater sonar image noise, and the effect of the traditional segmentation method is poor; Markov Model can accurately show the characteristics and relationship of image content. For segmentation algorithm of mattress image, using belief propagation (BP) algorithm .Experimental results show that it has a good effect.
\end{abstract}

Keywords-mattress sonar image; image segmentation; MRF model; belief propagation

\section{INTRODUCTION}

Sinking mattress is for preventing the riverbed's water and soil erosion; reducing the content of sediment in the river; and it is an important measure to prevent the local damage by the seepage of the riverbed and it's the foundation of waterway regulation work. The paving quality of sinking mattress is the direct influence of revetment engineering achievements, detection and tracking after arranging. On the one hand, it can provide the date for arranging to the future. On the other hand, it can find and handle problems after detecting problems. There are two kinds tracking monitoring method of previous sinking mattress lapping, the one is the artificial observation by underwater television, the other is by judging the sonar image gray texture and so on. This topic is based on the early stage of the equipment owned by the Yangtze River Waterway Bureau, changing the traditional artificial detection work, making full use of the combination of existing equipment and computer technology. Firstly, acquiring the underwater sonar image by siding scan sonar, generating the colorful RGB image ${ }^{[1]}$ by pseudo color technology and correcting the image, finally, processing image segmentation technology by graphic image and extracting the characteristic region of the image. The difference of underwater sonar image and natural image difference is that underwater sonar image is more and more noise, but the target image features relatively simple, so the traditional image segmentation methods as the classic Otsu algorithm ${ }^{[2]}$, the segmentation is low and sensitive to noise in sonar image is not suitable for processing, the Markov model can to overcome these problems, not only can describe the characteristics of the image exactly, but also can consider the relationship between the image neighborhood, this method modeling the local features of the image, establishing the

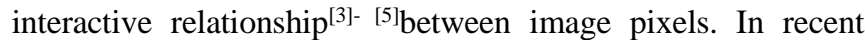
years, the MRF model has attracted much attention in image processing, and it growing maturity in vast majority of scholars' research, which is based on Maldives random field image segmentation method, it including local optimal relaxation algorithm, such as ICM algorithms of Fwuj ${ }^{[6]}$; the overall optimization, such as sampling algorithm of Gibbs ${ }^{[7]}$; efficient inferring algorithms, such as belief propagation ${ }^{[8]}$ and so on. Wang Lei used ICM algorithm in the study of side scan sonar image segmentation algorithm to segmentation and recognition the underwater fixed target ${ }^{[9]}$, although had made some achievements and ran fast, but the overall segmentation effect is poorer. The Gibbs sampling algorithm can obtain better segmentation result, but it takes a lot of time to find the global optimal solution, so it not applied to the sonar image. Along with the progress and improvement of all kinds of efficient reasoning algorithms, the advantages in the field of image segmentation are gradually reflected, application scenario for this article, Markov model sonar image can divide into two types: one part is the missed shadow area of sinking mattress lapping area, the other part is the texture background area of the paving area. Therefore, we just need put the image segmentation into two parts, using the algorithm of regional confidence propagation can shorten the elapsed time substantially. And according to this article's image segmentation algorithm of sinking mattress, on the one hand segmentation theory to reduce the time complexity of the algorithm, on the other hand, the characteristics of the image message co he strategy, two ways to overcome the belief propagation algorithm has relatively high shortcomings, segmentation takes time required greatly reduced. ${ }^{[10]-[15]}$

\section{MATTRESS SONAR IMAGE PREPROCESSING}

Based on the summary of research results and actual data, because we use a fixed ship to scan when side scan sonar scans, therefore the waves, gray image distortion caused by the removal of fish posture is not obvious. In this article, the main solution is to restore the geometric distortion of the sonar image. Geometric deformation refers to the sonar image and not strictly according to the proportion of record River factors are sinking mattress, the angle of scanning sonar, river geomorphology and so on. The main deformation is slant deformation and velocity distortion. Oblique distance refers to the deformation of side scan sonar transducer launch fanshaped sound waves to the river bottom, and receives the acoustic backscatter tilt direction under the scanning line on the sonar image reflects the slant distance transducer into the river, so the transverse proportion is not uniform, and cause the 
lateral deformation of target. Velocity distortion refers to the actual measurement, because the ship speed is different, leading to the sonar image, horizontal and vertical proportions are inconsistent. The traditional geometric deformation restoration is the slant range velocity distortion deformation, a separate correction, to eliminate the geometric deformation, but this has a lot of drawbacks, because of all kinds of deformation are mixed together, and it is difficult to eliminate. If improper methods, but also may cause new deformation. Based on the consideration of all kinds of deformation, this paper proposes a method of overall correction, the sonar image is projected to the actual geographical coordinates, on side scan sonar image homing, various deformation into consideration. The sonar image aspect ratio equal to eliminate geometric distortion. Specific methods are as follows: (1) tracking the river bottom and calculate the distance between tow fish and river bottom; (2) calculating the average of river bottom reversal point; (3) obtaining the track point coordinate; (4) calculating the coordinate of river bottom reversal point; (5) establishing a same proportion of $\mathrm{x}$ coordinate and y coordinate system and interpolation. Figure 1 shows the pre-processed mattress sonar image.

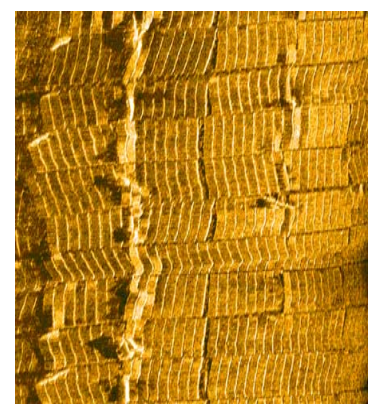

FIGURE I. PRE-PROCESSED MATTRESS SONAR IMAGE

\section{SEgMENTATION AND RECOGNITION OF NON- OVERLAPPING REGIONS OF THE SINK IMAGE}

\section{A. The Markov Model of the Mattress Image}

Automatic tracking and recognizing the non-overlapping area can be realized by segmentation and recognition of the mattress sonar image. Markov (MRF) random fields are widely used in the field of graphic image processing. The MRF model can not only describe the gray-change of the image properly, but also preserve the edge information of the image. So it has good robustness for detecting underwater noise. It is assumed that the characteristic observation field of the sonar image is $Y$, the pixel point is $\mathrm{y}$, the label field is $X$. In this paper, we only need to divide the mattress sonar image into two parts, one is the mattress laying area or mettress overlapping area, we mark it as $x_{1}$. The other part is the non-overlapping area, we mark it as $x_{2}$. According to the MAP criterion of the Markov random field model, the optimal segmentation of graphic image is to find the maximum a posteriori probability.

$$
P(X=x \mid Y=y)=\frac{P(Y=y \mid X=x) \cdot P(X=x)}{P(Y=y)}
$$

$P(Y=y \mid X=x)$ is a likelihood function, represents the conditional probability of the observed image $Y$ under a given marker $X . P(Y=y)$ is the prior distribution of the observed image. According to Hammersley-Clifford theorem, the Markov random field is equivalent to the Gibbs distribution:

$$
\mathrm{P}(\mathrm{X}=\mathrm{x})=\frac{1}{\mathrm{Z}} \cdot \exp (-\mathrm{E}(\mathrm{x}))
$$

$E(x)$ is the energy function, $\mathrm{Z}$ is a normalization constant. $Z=\sum \exp (-E(x))$. From formula (2), the maximum a posteriori probability problem is equivalent to minimizing the posterior energy function.

\section{B. Mattress Image Segmentation and Recognition}

Belief propagation (BP) algorithm is an efficient segmentation algorithm with good performance based on Markov random field, but the disadvantages is the running time of the algorithm will increased with the label field and pixel increasing .For this scenario we only need to split into two labels for the region and through the belief propagation algorithm and acceleration convergence strategy, we can further shorten the time of segmentation. According to the content of the last section, it is known that the optimal segmentation of the Markov random field is actually the maximum a posteriori probability, the maximum a posteriori probability problem is equivalent to the minimization of the energy function. Gibbs global energy function definition of BP algorithm is:

$$
\mathrm{E}(\mathrm{x})=\sum_{\mathrm{i}, \mathrm{j} \in \mathrm{N}} \mathrm{V}\left(\mathrm{y}_{\mathrm{i}}, \mathrm{y}_{\mathrm{j}}\right)+\sum_{\mathrm{i} \in \mathrm{I}} \mathrm{D}_{\mathrm{i}}\left(\mathrm{y}_{\mathrm{i}}\right)
$$

In the above formula, $E(x)$ is the global energy, $y_{i}$ represents the parallax value of point $i . D_{i}\left(y_{i}\right)$ represents the non-similarity measure of the parallax of point $i$ is $y_{i} . V\left(y_{i}, y_{j}\right)$ is a smoothing item, represents the discontinuous penalty, when $y_{i}$ and $y_{j}$ are assigned to $i$ and $j$ respectively. The larger the parallax, the greater the penalty. $N$ represents the set of four neighborhood points of all the pixels in the image. Traditional belief propagation algorithm message passing as shown below:

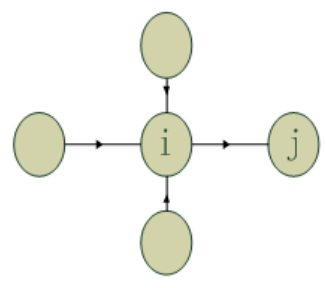

FIGURE II. MESSAGEPASSING OF BP ALGORITHM

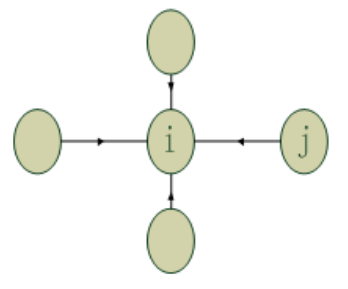

FIGURE III. BELIEF TRANSMISSION OF BP ALGORITHM
The message-passing formula is:

$$
\mathrm{m}_{\mathrm{i} \rightarrow \mathrm{j}}^{\mathrm{t}}\left(\mathrm{y}_{\mathrm{j}}\right)=\min \left(\mathrm{D}_{\mathrm{i}}\left(\mathrm{y}_{\mathrm{i}}\right)+\mathrm{V}\left(\mathrm{y}_{\mathrm{i}}, \mathrm{y}_{\mathrm{j}}\right)+\sum_{\mathrm{s}} \mathrm{m}_{\mathrm{s} \rightarrow \mathrm{i}}^{\mathrm{t}-1}\left(\mathrm{y}_{\mathrm{i}}\right)\right)
$$

$t$ is the number of iterations, $s$ represents the other nodes except $j$ in the adjacent nodes of $i . \sum_{s} m_{s \rightarrow i}^{t-1}\left(y_{i}\right)$ represents the sum of the information that $\mathrm{s}$ is passed to $\mathrm{i}$ during $t$-1-th iterations. Point $i$ of belief transmission process as shown in Figure 3. 
After $t$ times iterations, the message transmission tends to be stable.At this time, the belief of each point in the graph can be calculated by the transmission of belief. The formula is as follows:

$$
\mathrm{B}_{\mathrm{i}}\left(\mathrm{y}_{\mathrm{i}}\right)=\mathrm{D}_{\mathrm{i}}\left(\mathrm{y}_{\mathrm{i}}\right)+\sum_{\mathrm{s}} \mathrm{m}_{\mathrm{s} \rightarrow \mathrm{i}}^{\mathrm{t}}\left(\mathrm{y}_{\mathrm{i}}\right)
$$

For the sinking mattress layout of the image, it has a very obvious characteristic that its segmented regions of the image are relatively concentrated, so the region of the belief propagation algorithm can greatly reduce the time complexity of the algorithm. First, we define a local region energy model:

$$
e=\sum_{i, j \in n} V\left(y_{i}, y_{j}\right)+\sum_{i \in n} D_{i}\left(y_{i}\right)
$$

$e$ represents the energy in the region $n, n$ is a convergent pixel region, than the global energy $E$, The global energy minimization of the method is obtained by minimizing the local energy. The label of the local area is obtained from the minimum energy of the local area.

$$
\begin{gathered}
\mathrm{x}^{*}=\operatorname{arcmin} \mathrm{E}=\operatorname{arcmin} \\
\left(\sum_{\mathrm{i}, \mathrm{j} \in \mathrm{n}} \mathrm{V}\left(\mathrm{y}_{\mathrm{i}}, \mathrm{y}_{\mathrm{j}}\right)+\sum_{\mathrm{i} \in \mathrm{n}} \mathrm{D}_{\mathrm{i}}\left(\mathrm{y}_{\mathrm{i}}\right)\right)
\end{gathered}
$$

$x^{*}$ represents the label of the local area, in The above energy model, $D_{i}\left(y_{i}\right)=\lg P\left(y_{i} \mid x_{i}, \theta\right) . P\left(y_{i} \mid x_{i}, \theta\right)$ represents the conditional probability that $i$ belongs to label field $x_{i} . \theta$ is a set of $\left(\mu_{1}, \sigma_{1}^{2} \ldots \mu_{k}, \sigma_{k}^{2}\right), \mu_{k}, \sigma_{k}^{2}$ are the $k$-th Gaussian mean and variance, are calculate by using EM algorithm .The labeling process is described by a Gaussian mixture model:

$$
\begin{aligned}
\mathrm{P}(\mathrm{Y} \mid \mathrm{X}, \theta) & =\prod_{\mathrm{i}} \mathrm{P}\left(\mathrm{y}_{\mathrm{i}} \mid \mathrm{x}_{\mathrm{i}}, \theta\right) \\
& =\prod_{\mathrm{i}} \frac{1}{\sqrt{2 \pi \sigma_{\mathrm{k}}^{2}}} \exp \left(-\frac{\left(\mathrm{x}_{\mathrm{i}}-\mu_{\mathrm{k}}\right)^{2}}{2 \sigma_{\mathrm{k}}^{2}}\right)
\end{aligned}
$$

$V\left(y_{i}, y_{j}\right)=P\left(x_{i}, x_{j}, \beta\right), P\left(x_{i}, x_{j}, \beta\right)=-\beta \delta\left(x_{i}, x_{j}\right)$, and is a Interaction potential function, When $x_{i}=x_{j}, \delta\left(x_{i}, x_{j}\right)$ takes 0,otherwise, $\delta\left(x_{i}, x_{j}\right)$ takes 1 .

In this scenario, the segmentation of regional characteristics is relatively stable, after a certain number of iterations, the convergence of adjacent region labeling tends to be the same. The iteration in the iterative convergence region is of small significance, but it will consume a lot of time, so this paper adopts the following methods to reduce redundant computation. Assuming that in the $t$-th iteration, consistency labeling the pixel $i$ and adjacent $j$ node, the default regional converge, stop the iteration, skip the message passing between node $i$ and node $\mathrm{j}$, the default energy function value is minimum, $\mathrm{i}$ and $\mathrm{j}$ take the same label at the same time, then conduct the message passing of next node, otherwise continue the normal message iteration. Formula is as follows:

$$
\left|E^{t}(X)-E^{t-1}(X)\right| \leq \varepsilon
$$

The $\mathrm{E}^{\mathrm{t}}(\mathrm{X})$ represents the iteration energy of $t$-th times, $\mathrm{E}^{\mathrm{t}-1}(\mathrm{X})$ represents the $t-1$-th times, when the difference between two iterations is less than the minimum value epsilon, energy function has the minimum value at this time by default, because the cost of continuing disproportionate iteration disproportionately match the achievement. The algorithm steps are as following:

- Step 1: Setting up the MRF model of the sinking mattress image, and giving a priori model parameters $\beta$, the experiment results show that when $\beta=2$, the segmentation effect is the best;

- $\quad$ Step 2: With the maximum expectation (EM) algorithm to initialize $\mu_{\mathrm{k}}$ and $\sigma_{\mathrm{k}}^{2}$;

- $\quad$ Step 3: By 1, 2 initialization parameters segmentation image,

$$
\mathrm{X}^{\mathrm{p}}=\operatorname{arcmax} \mathrm{P}\left(\mathrm{X} \mid \mathrm{Y}, \beta, \mu_{\mathrm{k}}, \sigma_{\mathrm{k}}^{2}\right)
$$

- $\quad$ Step 4: For $i=1$ To N, if node $y_{i}$ and node $y_{j}$ label field belongs to the same label, message passing directly to the next node, otherwise $y_{i}$ compute nodes of local energy, updating the local message passing, according to the MAP criterion to estimate the $\mathrm{y}_{\mathrm{i}}$ label $\mathrm{x}_{\mathrm{i}}^{\mathrm{n}}$;

- $\quad$ Step 5: Calculating the global energy $\mathrm{E}^{\mathrm{t}}(\mathrm{X})$, if

$\left|E^{t}(X)-E^{t-1}(X)\right| \leq \varepsilon$, the distribution of the label field, that is the sink image segmentation results, or returnto the fourth step to continue the iteration until the final segmentation results.

Running this algorithm, if there is no overlapping region that is the label field for the river area, so that the mattress laying work is not qualified, and need for timely repair.

\section{ANALYSIS OF EXPERIMENTAL RESULTS}

By comparing the three image segmentation results and analyzing this result, the experimental environment is Matlab, select a few sinking mattress images of no lapping area, the image' size is 200X220, Figure 4-1a is the amplifying sonar image of sinking mattress detail, Figure $4-1 b$ is a normal sonar image. Figure 4-2a and 4-2b are the images of classic natural images segmentation method OTSU algorithm. Obviously, the experimental results show that after a series of dying treatments, many noise points still exist in the image, it has a great effect on the image segmentation results, so the method of sonar in underwater image processing is inapplicable. Figure 4-3a and Figure 4-3b is a ICM image segmentation method based on Markov random fields, the widely used method of target recognition in the scene in the water, but only a limited effect for the identification of fixed objects, the sinking mattress image recognition area is not fixed, the segmentation result is not ideal. Figure $4-4 a$ and Figure $4-4 b$ is after the BP segmentation algorithm, recognition of the sinking mattress separation area, two kinds of method mentioned above, the recognition area is closest to the original image, the influence of noise is very little, edge contour is clear, local image features is obvious, segmentation results is accurately. 


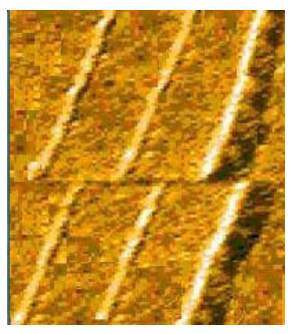

FIGURE IV-1A. AMPLIFYING SONAR IMAGE OF SINKING MATTRESS DETAIL

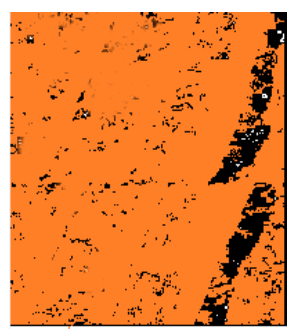

FIGURE IV-2A. IMAGE SEGMENTATION BY OTSU ALGORITHM

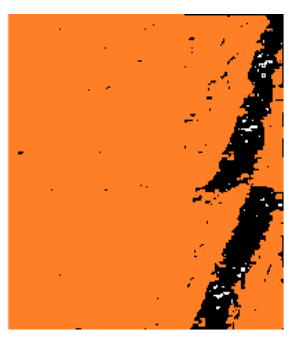

FIGURE IV-3A. IMAGE SEGMENTATION BY ICM ALGORITHM

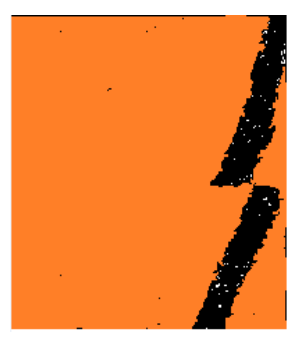

FIGURE IV-4A. IMAGE SEGMENTATION BY BP ALGORITHM

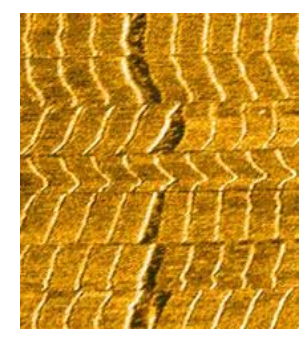

FIGURE IV-1B. NORMAL SONAR IMAGE

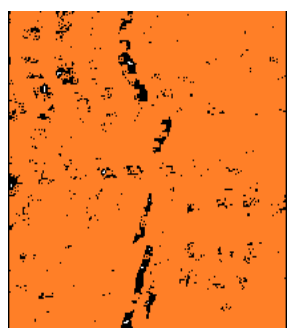

FIGURE IV-2B. IMAGE SEGMENTATION BY ICM ALGORITHM

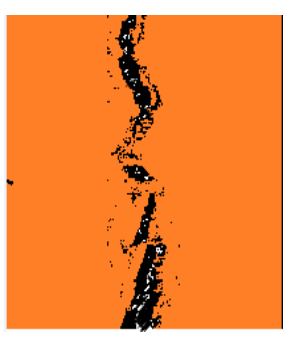

FIGURE IV-3B. IMAGE SEGMENTATION BY ICM ALGORITHM

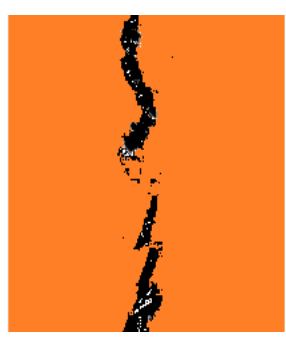

FIGURE IV-4B. IMAGE SEGMENTATION BY BP ALGORITHM

TABLE I. THREE KINDS OF IMAGE SEGMENTATION TIME COMPARISON TABLE

\begin{tabular}{cccc}
\hline algorithm & $\begin{array}{c}\text { Otsu } \\
\text { algorithm }\end{array}$ & $\begin{array}{c}\text { ICM } \\
\text { algorithm }\end{array}$ & $\begin{array}{c}\text { BP } \\
\text { algorithm }\end{array}$ \\
\hline Figure a & $64 \mathrm{~ms}$ & $96 \mathrm{~ms}$ & $103 \mathrm{~ms}$ \\
Figure b & $89 \mathrm{~ms}$ & $108 \mathrm{~ms}$ & $113 \mathrm{~ms}$ \\
\hline
\end{tabular}

It can be known from the above table, although the $\mathrm{BP}$ algorithm in this paper is more time-consuming than the Otsu

algorithm, But the segmentation effect is much better than the Otsu algorithm. Compared with the ICM algorithm, Consuming slightly longer, but compared with the segmentation effect, the segmentation of this algorithm is better. Obviously, the misclassification rate of $\mathrm{BP}$ algorithm is the lowest.

\section{CONCLUSION}

Nowadays, more and more advanced channel detection equipment, sonar image processing technology is becoming more and more mature, it brought more and more convenient processing method to channel detection work. In this article, the extraction method of belief propagation algorithm has better segmentation effect advantage, improved the disadvantages of long segmentation time in the scene, greatly shorten the time for segmentation and recognition, accurate identification of the sinking mattress separation area, good experimental results are achieved in this scenario, the detection of sinking tracking the work provides the basis for accurate judgment. The next task is to research how to distinguish the overlapping width of the mattres.

\section{REFERENCES}

[1] Zhang Qun. Research on underwater sonar imaging technology and enhancement methods in deep water sinking mettress [J]. Waterway and Harbor, 2014 (3): 271-276.

[2] Zhang xinming, Sun Yingyu, Zheng Yanbing. Otsu image segmentation based on two dimensional histogram and its fast implementation[J]. Electronic journal, 2011, 39(8):1778-1784.

[3] Ding Liang, Zhang Yongping, Zhang Xueying. Review of image segmentationmethods and performance evaluation [J]. Software, 2010, 31 (12): 78-83.

[4] Li Xuchao, Zhu Shanan. A review of Markov random field method in image segmentation[J].Chinese Journal of image and graphics, 2007, 12 (5): 789-798.

[5] Kato Z, Pong T C. A Markov random field image segmentation model for color textured images[J]. Image \& Vision Computing, 2006, 24(10):1103-1114.

[6] Fwu J, Djuric P M. Unsupervised vector image segmentation by a tree structure-ICM algorithm.[J]. IEEE Transactions on Medical Imaging, 1996, 15(6):871-80.

[7] Kato Z. Segmentation of color images via reversible jump MCMC sampling[J]. Image \& Vision Computing, 2008, 26(3):361-371.

[8] Sun B J, Zheng N N, Shum H Y. Stereo Matching using Belif Propagation[C]// IEEE Transactions on Pattern Analysis and Machine Intelligence. 2010.

[9] Wang Lei. Research on image segmentation algorithm of side scan sonar[D]. Harbin Engineering University, 2013.

[10] Potetz B, Tai S L. Efficient belief propagation for higher-order cliques using linear constraint nodes[J]. Computer Vision \& Image Understanding, 2008, 112(1):39-54.

[11] Liu Guanghui, Ren Qingchang Meng Yuebo. 2014, 31 (4): 1232-1235.

[12] Jianhua, JIAO, Licheng, et al. Image Segmentation via Mean Shift and Loopy Belief Propagation[J]. Wuhan University Journal of Natural Sciences, 2010, 15(1):43-50.

[13] Yedidia J S, Freeman W T, Weiss Y. Understanding belief propagation and its generalizations[J]. Exploring Artificial Intelligence in the New Millenium, 2003, 54(1):276-286.

[14] Gormley M R, Eisner J. Structured Belief Propagation for NLP[C]// Tutorials. 2015.

[15] Felzenszwalb P F, Huttenlocher D P. Efficient Belief Propagation for Early Vision[J]. International Journal of Computer Vision, 2006. 